\title{
A New Model and a Hyper-heuristic Approach for Two-dimensional Shelf Space Allocation
}

\author{
Ruibin Bai ${ }^{1}$, Tom van Woensel ${ }^{2}$, Graham Kendall ${ }^{3}$ and Edmund K. Burke ${ }^{4}$ \\ 1 Department of Computer Science, University of Nottingham Ningbo China, 199 Taikang East Road, Ningbo, China, 315100, \\ Tel. +86-574-88180278, Fax +86-574-88180125. e-mail: ruibin.bai@nottingham.edu.cn. \\ 2 School of Industrial Engineering, Technische Universiteit Eindhoven, Den Dolech 2, Pav. F05, Eindhoven NL 5600 MB, The \\ Netherlands. e-mail: t.v.woensel@tue.nl. \\ 3 School of Computer Science, University of Nottingham, Nottingham, UK and Semenyih, Malaysia. \\ e-mail: graham.kendall@nottingham.ac.uk \\ 4 Department of Computing Science and Mathematics, University of Stirling, Cottrell Building, Stirling, FK9 4LA, UK. e-mail: \\ e.k.burke@stir.ac.uk
}

Received: September 2011 / Revised version:

\begin{abstract}
In this paper, we propose a two-dimensional shelf space allocation model. The second dimension stems from the height of the shelf. This results in an integer nonlinear programming model with a complex form of objective function. We propose a multiple neighborhood approach which is a hybridization of a simulated annealing algorithm with a hyper-heuristic learning mechanism. Experiments based on empirical data from both real-world and artificial instances show that the shelf space utilization and the resulting sales can be greatly improved when compared with a gradient method. Sensitivity analysis on the input parameters and the shelf space show the benefits of the proposed algorithm both in sales and in robustness.
\end{abstract}

Keywords: shelf space allocation; two-dimensional; retail; multi-neighborhood search; hyper-heuristics 


\section{Motivation}

Retailers aim to maximize availability of the goods in their product line at a minimum cost to operations. In the stores, these goals have to be realized on the shelves, where any product meets the customer. Shelf spaces are expensive resources for many retail stores and supermarkets. Space planning and shelf operation is thus a major area that can significantly improve a retailer's financial performance and customer satisfaction.

One of the responsibilities of marketing is designing an attractive presentation on the shelves in order to attract customers. The amount of shelf space allocated to a product is thus primarily a consequence of marketing decisions: i.e. the merchandizing category to which the product is assigned and the allocated number of facings (the number of slots on the front of a retail shelf). Planograms (Bai and Kendall, 2005) represent plans of how retail products will be laid out on the shelves, and show retailers where and how each Stock Keeping Unit (SKU) should be displayed. SKUs are used to uniquely identify a specific product or goods in location and its characteristics. It is the smallest management unit in a retail store. Planograms determine the available shelf space for the operations. From both an operations and a marketing point of view, it is thus important to produce high-quality planograms.

A planogram contains important information for the execution of the operations. In general, when constructing planograms the retailer decides on the assortment composition (which items are in the assortment?), the location of the item in the stores (where are the aisles, section, and shelf locations?) and the amount of space allocated to each item (how many facings and consumer units?). In an empirical study by Van Woensel et al. (2006), it was observed that planogram integrity is a serious issue for retailers. Planogram integrity is the degree to which the planograms are followed in practice. The empirical evidence clearly demonstrated that the majority of differences are mainly due to facing differences. In second and third place of importance, it was found that assortment and location differences are important. A major consequence of a lack of planogram integrity is the loss of a substantial level of efficiency both in terms of the marketing strategy as well as in the operational executions.

An important underlying driver for these planogram integrity problems, could be related back to the inability of the planogram tools to cope with real-life situations. The store managers tried to remedy this by taking into account a greater number of factors and changing the proposed planograms. In general, the motivation relates back to the issue that planograms are mainly built based on marketing decision rules (e.g. the number of facings per product category) and are often one-dimensional (i.e. do not take into account the depth nor height of the shelves). This paper takes a significant step towards more efficient planograming tools. In this paper, we propose to model shelves as two-dimensional spaces, whilst considering the location 
effects of shelf space on the demand. Moreover, we proposed an efficient hyper-heuristic method for tackling this two-dimensional plangram problem.

The paper makes the following contributions:

- Most of the shelf space allocation models discussed in the literature treat shelf space one-dimensionally, quantified either in volume or in shelf length. That is, the capacity of shelf is usually quantified as a single real value (see e.g. (Corstjens and Doyle, 1981; Dreze et al., 1994; Urban, 1998; Yang, 2001; Hwang et al., 2005; Bai and Kendall, 2008)). These models are mainly useful if all the shelves and items concerned have similar height dimensions and it is impossible to improve the space utilization by manipulating items across shelves. These models are also useful when items cannot be stacked for various reasons (for example wine and milk bottles). Our paper presents a 2-dimensional approach taking into account not only shelf length but also shelf height. In addition, the paper proposes a hyper-heuristic based approach which can potentially handle large-sized problem instances.

- Today, many items can be stacked together in order to make full use of the shelf space with regard to the height dimension, but this is not explicitly considered in current planogram optimization tools. Of course, in practice shelf operators can still stack items on top of the facings allocated according to a one-dimensional model. However, this usually leads to decreased space utilization and productivity. We show, via a rigorous scale of experiments, that explicitly taking into account the height dimension will improve the space utilization.

The paper is structured as follows: Section 2 discusses related work. Section 3 formulates our model of the shelf space allocation. Methodologies that are used to optimize the problem model are presented in Section 4. Numerical examples are presented in Section 6 along with sensitivity analysis on parameter estimation errors and Section 7 concludes the paper.

\section{Related Work}

The study of shelf space allocation dates back to the 1960's with some empirical experiments being carried out to study the influence of shelf space operations. For example, Kotzan and Evanson (1969) made empirical studies for three products from eight chain stores and found that a significant relationship existed between shelf facings and sales. Cox (1970) carried out similar experiments for products from two brands of two categories, salt and coffee cream. He reported a very weak relationship between shelf facings and sales.

Space elasticity has been widely used to measure the responsiveness of the sales with regards to the change of allocated space. Curhan (1972) defined space elasticity as "the ratio of relative change in unit sales 
to relative change in shelf space" and reported an average value of 0.212 . However, this is just an average value: the actual value of the space elasticity can be very different, depending on the products, stores and instore layout (Curhan, 1973). Cross-product effects were also investigated in (Dreze et al., 1994) where space manipulations were made to enhance complementary shopping by placing complementary products together. The results showed that complementary merchandizing resulted a positive boost in sales (over 5\%) on the tested products (toothbrush, toothpaste and laundry care). It was also found that, compared with shelf facings, location had a larger impact as long as a minimum number of shelf facings (to avoid out-of-stocks) was maintained. Shelves at the eyesight level are more favorable than shelves located at the top or bottom of the shelf fixtures. Quantitative models have been proposed to describe the relationship between shelf space and sales. Corstjens and Doyle (1981) firstly formulated their model in a non-linear multiplicative way and incorporated cross elasticities, a set of problem parameters that reflect the interrelationships between the different products under consideration. The inventory and handling cost effects were also considered. Two parameter estimation approaches were also compared and discussed. Zufryden (1986) proposed a dynamic programming model for the shelf space allocation problem, which also took into account some non-space factors such as price, advertising, promotion, store characteristics, etc. However, the approach may only be suitable for small sized problems and becomes computationally expensive for larger problem instances.

Recognizing that shelf space allocation is also closely related with other retailing problems, some integrated models have also been proposed. For example, Borin et al.'s model (Borin et al., 1994) could simultaneously provide a solution for both product assortment and shelf space allocation. A simulated annealing algorithm was used to optimize the proposed model. Urban (1998) integrated product assortment and inventory control with a traditional shelf space allocation model. A genetic algorithm was proposed to solve the problem. However, these models usually involve a large number of parameters. Obtaining a reliable estimation of these parameters is generally challenging and time-consuming. Therefore, it is difficult to put these models into a real application. A simplified linear model was proposed in (Yang, 2001), which considered the location effect of shelves on sales. The model, in fact, is a varied type of bounded multi-knapsack problem, which is generally difficult to solve. The authors designed several greedy heuristics to optimize the problem model.

However, these simple heuristics will only return a sub-optimal solution in many cases due to their inability to escape from local optimality. To solve the weakness of these simple heuristics, Lim et al. (2004) proposed several approaches, including a network flow procedure, tabu search and a modified squeaky-wheel algorithm. Experimental results, over several problem instances, showed that the modified squeaky-wheel algorithm is able to obtain a large improvement over the simple greedy heuristics used in (Yang, 2001). Recently, another 
shelf space allocation model was proposed in (Hwang et al., 2005) which considers both the inventory and location influence on the demand. Both a gradient-based procedure and a genetic algorithm were used to solve the problem and experimental results on several small numerical instances showed that the genetic algorithm is able to obtain superior results. Bai and Kendall (2008) proposed an integrated inventory and shelf space allocation model specifically to handle fresh produce. A modified generalized reduced gradient approach was used to search for good quality solutions. Murray et al. (2010) and Hwang et al. (2009) have presented shelf space allocation models related to the one presented in our paper. Specifically, Hwang et al. (2009) looked into a simultaneous shelf space design and allocation problem. A mathematical model was developed and two solution procedures were proposed based on a genetic algorithm (Goldberg, 1989; Sastry et al., 2005). This was applied to some very small test instances of four products. Murray et al. (2010) developed a model that jointly optimizes a retailer's decisions for product prices, display facing areas, display orientations and shelfspace locations in a product category. This comprehensive model is solved by branch-and-bound procedures on some small test instances. Our paper is distinct by the use of hyper-heuristics and the ability to solve larger, more realistic sized instances.

Hyper-heuristics (Burke et al., 2003a; Ross, 2005) have recently received increasing attention partly because one of their motivating goals is to establish a higher level of generality across different problem solving scenarios. A hyper-heuristic searches across a space of heuristics rather than the more usual heuristic approach of dealing with the solution space directly. Hyper-heuristics have successfully been applied to several difficult search problems, including timetabling and rostering (Burke et al., 2003b; Bai et al., 2012; Burke et al., 2006, 2007), scheduling (Hart et al., 1998), and packing and layout optimization (Bai et al., 2008; Dowsland et al., 2007).

\section{Model Development}

In a similar way to that presented in (Yang, 2001), it is assumed that retailers can prevent going out-of-stock by improving their supply chain. Space allocation is carried out within a given category (space allocation between different categories is usually a strategic problem rather than a quantitative one). Shelves are in multi-level with each level having a different impact factor. The problem can be formulated as follows: we are given a number of shelves $m$ with each shelf $j$ having a demand impact factor $\gamma_{j}\left(\gamma_{j} \geq 1\right)$ and we are also given a number of $n$ SKUs. The least popular shelves (i.e. either the bottom or the top shelves) have an impact factor of 1 and shelves that are close to "eye-level" have $\gamma_{i}$ values greater than 1 . An SKU can be stacked on the top of another but is limited to being stacked within the same SKU. The problem is to 
allocate an appropriate number of facings to each SKU $i$ such that the total sales of the SKUs are maximized.

The notations used in the model are defined in Table 1.

Table 1. List of variables used in the analysis

\begin{tabular}{ll}
\hline & Description \\
\hline$L_{j}$ & Length of shelf $j$ \\
$H_{j}$ & Height of shelf $j$ \\
$l_{i}$ & Length of SKU $i$ \\
$h_{i}$ & Height of SKU $i$ \\
$\pi_{i j}$ & Stack coefficient of item $i$ on shelf $j$, and $\pi_{i j}=\left\lfloor\frac{H_{j}}{h_{i}}\right\rfloor$ \\
$F_{i}$ & Demand function of item $i$ over time \\
$M_{i}$ & Sales realized on item $i$ \\
$\alpha_{i}$ & Scale parameter for demand function of item $i$ \\
$\beta_{i}$ & Space elasticity for item $i$ \\
$\beta_{i k}$ & Cross elasticity of item $k$ on item $i$ and $\beta_{i k}$ is asymmetric. \\
$\gamma_{j}$ & Location impact factor of shelf $j$ \\
$p_{i}$ & Unit selling price of item $i$ \\
$s_{i}^{\text {min }}$ & Minimum number of facings for item $i$ \\
$s_{i}^{\text {max }}$ & Maximum number of facings for item $i$ \\
$x_{i j}$ & length facing of shelf $j$ allocated to item $i$ \\
$x_{i}$ & Total shelf length facings allocated to item $i$, i.e. $x_{i}=\sum_{j=1}^{m} x_{i j}$ \\
$s_{i}$ & Total facings allocated to item $i$ \\
$y_{i j}$ & $y_{i j}=1$, if item $i$ is placed on shelf $j$ \\
& $y_{i j}=0$, if item $i$ is not placed on shelf $j$ \\
$z_{i}$ & Shelf ID on which product $i$ is displayed \\
\hline &
\end{tabular}

For simplicity, we define length facing as the number of facings that are allocated to an item along the shelf length and stack coefficient as the maximum number of facings that can be allocated in height. Therefore, the total number of facings of an item is the product of length facings and the stack coefficient.

We use the same deterministic demand function proposed by (Hwang et al., 2005). In their paper, they formulated the demand function as a multiplicative form of direct space effect, cross space effect and location effect:

$$
F_{i}=\alpha_{i} \times s_{i}^{\beta_{i}} \times\left[\prod_{k \neq i}^{n} s_{k}^{\beta_{i k}}\right] \times \Upsilon_{i}
$$

where $s_{i}$ is the total number of facings allocated to item $i$. For a stacking coefficient value $\pi_{i j}, s_{i}$ can be calculated as

$$
s_{i}=\sum_{j=1}^{m} x_{i j} \times \pi_{i j}
$$


and $\Upsilon_{i}$ the average location effect obtained as

$$
\Upsilon_{i}=\frac{\sum_{j=1}^{m} x_{i j} \pi_{i j} \gamma_{j}}{s_{i}}
$$

The model can then be formulated as follows

$$
\max \sum_{i=1}^{n} M_{i}=\sum_{i=1}^{n} p_{i} F_{i}
$$

subject to:

$$
\begin{aligned}
\sum_{i=1}^{n} l_{i} x_{i j} & \leq L_{j}, \forall j \\
y_{i j} & \leq \pi_{i j}, \forall i, j \\
s_{i}^{\min } & \leq s_{i} \leq s_{i}^{\max }, \forall i \\
x_{i j} & \in\{0\} \cup Z^{+}, \forall i, j \\
y_{i j} & \in\{0,1\}, \forall i, j \\
y_{i j} & \leq x_{i j}, \forall i, j \\
y_{i j} \times L_{j} / l_{i} & \geq x_{i j}, \forall i, j \\
\sum_{j=1}^{m} y_{i j} & =1, \forall i
\end{aligned}
$$

Constraints (5) and (6) make sure that the items allocated to each shelf do not exceed the capacity of the shelf (both in length and height). Constraint (7) defines the minimum and maximum facings which can be allocated to each item. Constraints (8), (9), (10) and (11) define the integrality of decision variables $x_{i j}$ and $y_{i j}$ and their relationships. Constraint (12) is a cluster constraint which ensures that the same type of items have to be displayed together on one shelf. The cluster constraint is not considered in previous models, which means that the same type of items can be displayed on several different shelves. In practice, retailers usually want to keep the same type of items in the same place to present a large attractive block. Therefore, this constraint is included in our model.

To illustrate the necessity to use a 2D model, we now consider one of the 1D numerical examples presented in (Hwang et al., 2005). The example is drawn from the Dataset1 with 4 "mixed" items (i.e. including both substitutive and complementary items) and 4 shelves. For completeness, the details of the problem instance are also presented in Table 2. 
Table 2. A numerical example taken from (Hwang et al., 2005)

\begin{tabular}{cccccccccc}
\hline & & & & & & \multicolumn{3}{c}{$\beta_{i k}$} \\
\cline { 6 - 10 } item $_{i}$ & $p_{i}$ & $l_{i}$ & $s_{i}^{\min }$ & $s_{i}^{\max }$ & $\alpha_{i}$ & 1 & 2 & 3 & 4 \\
\hline 1 & 5.89 & 1 & 1 & 6 & 62.26 & 0.500 & 0.011 & -0.014 & -0.010 \\
2 & 4.37 & 1 & 1 & 6 & 70.11 & 0.010 & 0.250 & -0.009 & 0.014 \\
3 & 4.91 & 1 & 1 & 6 & 66.92 & -0.005 & -0.006 & 0.400 & -0.011 \\
4 & 6.14 & 1 & 1 & 6 & 61.22 & -0.008 & 0.009 & -0.015 & 0.340 \\
\hline \multicolumn{2}{l}{ Shelf data: $m=4, L_{j}=\{3,3,3,3\}, \gamma_{j}=\{1.3,1.2,1.1,1.0\}$} \\
\hline
\end{tabular}

The optimal solution (obtained by an exhaustive search procedure) for this 1D problem instance is displayed in Fig. 1 (a). As can be seen, this example assumes that both shelves and items have unit height sizes. In reality, however, shelves are often in different heights (ranging from 100mm to $1000 \mathrm{~mm}$ based on our own data) and items can be stacked if the shelf space and other conditions allow it. Now suppose that the fourth shelf is two units in height instead of one. If the 1D planogram solution is still used, one would probably come up with a solution by stacking three more of item 2 onto the fourth shelf (corresponding to Fig. 1 (b)). This solution gives an objective value of 2492.55. However, the true optimal solution is 2616.29 which is shown in Fig. 1 (c). The retailer could potentially lose 123.74 worth of sales in this case. This simple example shows that using a 1D planogram model can lead to considerable losses. It should be noted that, in this example, we only slightly changed the original data instance (one shelf only). In practice, both shelves and items may be of a different height, which justifies the necessity of using a two-dimensional planogram model, although item heights are not critical: (1) if the shelves are of the same height, the problems with items of different heights could be easily transformed into a one-dimensional problem; (2) even if the items are of the same height, the problems with shelves of different heights can be transformed into a one-dimensional problem.

\section{Solution Approaches}

The problem presented in (4) subject to (5)-(12) is an integer nonlinear programming model with a complex form of objective function. The decision variables are $x_{i j}$. Due to the cluster constraint (12), each item can only be displayed on one shelf. Therefore, the solution can be represented by the following two vectors: let $z_{i}$ be the shelf ID on which item $i$ is displayed. Let $x_{i}$ be the shelf length facings that are allocated to item $i$. This reduces the number of decision variables to $2 n$. Given this, the problem is still very difficult to solve in a closed form. A complete search algorithm is also prohibitive even for a medium sized problem. We therefore turn our attention to heuristic approaches. This is also supported in the literature where heuristic 


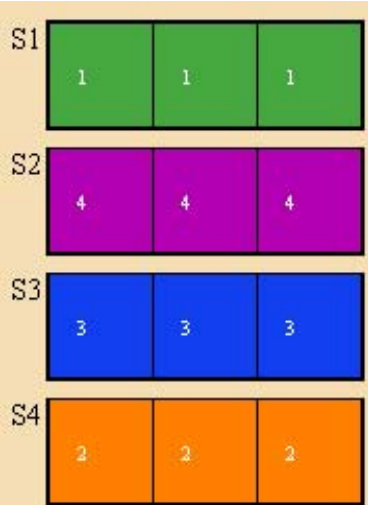

(a)

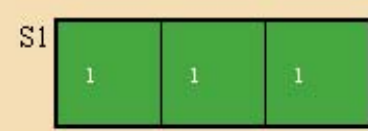

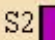

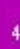

S3
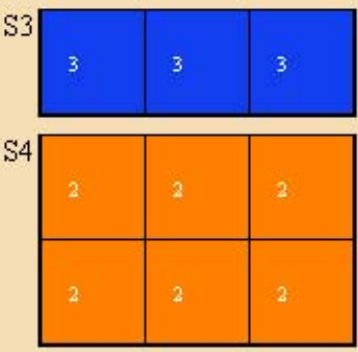

(b)

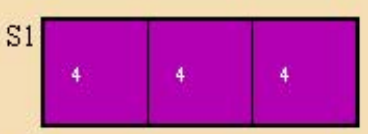

$\mathrm{S} 2$
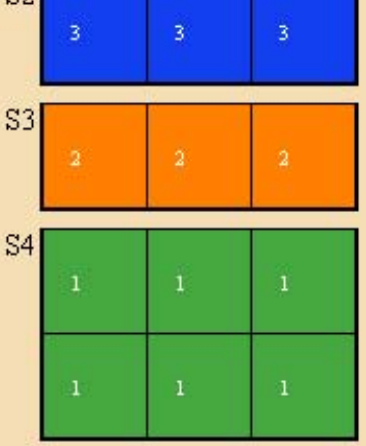

(c)

Fig. 1. A comparison of a 1D planogram with a 2D planogram (a) Optimal solution for the 1D planogram. (b) the 1D planogram when shelf height changed. (c) Optimal solution of the 2D planogram

approaches have been widely used to solve several shelf space allocation or related problems (Borin et al., 1994; Urban, 1998; Yang, 2001; Lim et al., 2004; Hwang et al., 2005; Bai et al., 2008).

\subsection{A gradient approach}

Because of the limited shelf space resources, items compete against each other for space. A gradient approach iteratively allocates shelf space to the items that can produce the largest reward per unit space in terms of the objective value (in this case, sales). However, due to the nonlinearity of the objective function, the ratio of sales to the allocated space varies with different shelf facing values $s_{i}$. The partial derivative of the objective function with regard to decision variables $x_{u v}(u=1, . . n, v=1, \ldots, m)$ is quite complex and can be presented as follows:

$$
\begin{aligned}
\frac{\partial \sum_{i=1}^{n} M_{i}}{\partial x_{u v}}= & \sum_{i \neq u}\left[p_{i} \alpha_{i} \beta_{i u} \pi_{u v} s_{i}^{\beta_{i}-1} s_{u}^{\beta_{i u}-1} \prod_{k \neq i, u} s_{k}^{\beta_{i k}} \sum_{j=1}^{m}\left(x_{i j} \pi_{i j} \gamma_{j}\right)\right]+ \\
& p_{u} \alpha_{u} \pi_{u v} s_{u}^{\beta_{u}-2} \prod_{k \neq u} s_{k}^{\beta_{u k}}\left[\left(\beta_{u}-1\right) \sum_{j=1}^{m}\left(x_{u j} \pi_{u j} r_{j}\right)+s_{u} \gamma_{v}\right]
\end{aligned}
$$

Due to the minimum facing constraint (7), most constructive heuristics for shelf space allocation problems adopt a two-phase procedure, which we also use in this paper. The first phase of the algorithm tries to allocate 
space to each item so that the minimum facing requirements are satisfied. In the second phase, the algorithm then repeatedly allocates the remaining space to the most preferable items according to some criteria. For example, the partial derivative function (13) would be a good candidate for this purpose. We can simply repeatedly increase the value of $x_{u v}$ that gives the largest partial derivative value according to function (13), assuming there is still sufficient space available on shelf $v$. If this is not the case, the second largest partial derivative value is chosen, and so on. However, there is a problem. The existence of the cluster constraint (12) means that each item can only be displayed on one of the shelves. Therefore, once the first facing of an item is allocated to a given shelf, the remaining facings of this item have to be on the same shelf. This means that the first phase of the procedure is paramount in terms of the accommodating shelf for each item. If function (13) is adopted directly in the first phase of the algorithm, the algorithm tends to allocate the first facing of most of items to the most attractive shelves and leaves some of the less attractive shelves empty. To solve this problem, the gradient algorithm used in this paper selects shelves based on the following rules, instead of function (13). At each iteration, the shelf with largest residual length is selected, with ties broken by favoring larger shelf heights and then the location impact factor.

The algorithm can be described as follows:

\section{S1. Initial Phase}

S1.1 Select a shelf $v$ with the largest residual capacity, with ties broken by favoring larger shelf height and then the larger location coefficient.

S1.2 For each item $u$ that has not been initialized, set $s_{u}=s_{u}^{\min }, x_{u}=\left\lceil s_{u} / \pi_{u v}\right\rceil, s_{u}=x_{u} \times \pi_{u v}, z_{u}=v$.

S1.3 Calculate the partial derivative according to function (13), select the item that has the largest partial derivative value and that can be accommodated by the shelf $v$. Label this item to be initialized.

S1.4 If all items are initialized, go to S2, otherwise, go to S1.1.

\section{S2. Iterative Phase}

S2.1 For each item and its accommodating shelf, update the corresponding derivative value according to function (13). Sort the items according to the descending order of their derivative values.

S2.2 Select the first item $u$ in the list, denote $v$ be the shelf on which $u$ is placed, set $x_{u}=x_{u}+1$, $s_{u}=s_{u}+\pi_{u v}$. If this results in an infeasible solution, set $x_{u}=x_{u}-1, s_{u}=s_{u}-\pi_{u v}$ and exclude this item from further consideration.

S2.3 Stop if no more facings can be allocated to any shelf, otherwise go to S2.1. 


\subsection{A Multi-neighborhood Approach}

Metaheuristics (Glover and Kochenberger, 2003) have been widely used to tackle highly constrained optimization problems from various fields. Multiple neighborhood search approaches have recently emerged as a popular meta-heuristic technique because of their ability to handle difficult constraints and obtain high quality solutions. Utilizing multiple neighborhoods could increase the accessibility of the search space and also improve the efficiency of the local search.

In this paper, a hybrid multiple neighborhood approach is proposed to solve this two-dimensional shelf space allocation problem. The multiple neighborhood approach utilizes a collection of neighborhoods in hybridization with a simulated annealing algorithm and a hyper-heuristic (Burke et al., 2003a; Ross, 2005) learning mechanism. This approach has shown considerable generality and competitiveness across large number of datasets of two very different combinatorial optimisation problems (bin packing and university course timetabling) (Bai et al., 2012). The increased generality is achieved by preventing hyper-heuristic from using problem-dependent information. Fig. 2 gives a pseudo-code of this approach. Based on a given initial solution and a set of neighborhoods, this hyper-heuristic approach intelligently changes the neighborhood preferences during the search and the simulated annealing is used to determine whether a given neighborhood move suggested by the hyper-heuristic is accepted or rejected. More specifically, each neighborhood is associated with a weight $w_{i}$ to represent its preference in comparison to the other neighborhoods. At each iteration, a neighborhood is stochastically ranked by the probability $p_{i}=w_{i} / \sum_{i=1}^{n} w_{i}$. Initially, weights are set to a predefined common minimum $w_{\min }$ and then updated periodically (defined by a learning period, or LP). Performance of each neighborhood in a given learning period is monitored and measured by two criteria, namely acceptance ratio and percentage of new solutions being generated. During normal "annealing" phase, the weight $w_{i}$ is set to the acceptance ratio of each heuristic in the previous period and then stays unchanged during the learning period. If, however, at the end of the period the average acceptance ratio among all neighborhoods falls below a threshold, a "reheating" strategy is triggered to diversify the search and neighborhoods that are more likely to generate new solutions are favoured. Interested readers could refer to (Bai et al., 2012) for more discussions about this approach and its relationship with some of other metaheuristics, for example, iterated local search, variable neighbourhood search and adaptive large neighborhood search approaches.

\subsubsection{Neighborhood structures The following neighborhood structures are used.}


N1 Swap: This neighborhood includes all the neighboring solutions that can be generated by swapping one shelf length facing of two items $i, k$ that are sharing the same shelf in the current solution. i.e. $x_{i}=x_{i}+1$, $x_{k}=x_{k}-1$.

N2 Shift: This neighborhood aims to improve the current solution by changing an item's current accommodating shelf. The neighborhood consists of all the solutions that can be generated by moving all the facings of an item from its current shelf to a different shelf.

N3 Interchange: This neighborhood enables the algorithm to interchange two items' $(i, k)$ accommodating shelves if they are different (i.e. $z_{i} \leftrightarrow z_{k}$ ). This neighborhood is different from N1 in that it operates on the facings of items from two different shelves while N1 operates on the items' facings on the same shelf.

N4 Add_Facing: This neighborhood consists of all the neighboring solutions that can be generated by increasing the length facing $x_{i}$ of item $i$ by one.

N5 Delete_Facing: This neighborhood function deletes a length facing of a randomly selected item.

4.2.2 Constraint handling Since not all of the above neighborhood moves produce feasible solutions, a mechanism has to be used to ensure that the algorithm searches within feasible regions of the search space. In this paper, the following method is used. For neighborhoods N1, N4 and N5 that involve adding/deleting facings to/from a single shelf, if a neighborhood move leads to an infeasible solution, the move is rejected and the search goes back to the previous point. For neighborhoods N2 and N3, if the solution is not feasible because of violations of shelf length constraint (6), the length facing variables $x_{i}$ that are involved in the moves are adjusted to their maximum possible values while attempting to satisfy all the constraints. If this fails to generate a feasible solution, the current neighboring solution is discarded and another neighboring solution is examined. The complete multiple neighborhood search algorithm is shown in Fig. 2.

In this application, the parameters of the multi-neighborhood algorithm are set as follows: the initial and stopping non-improving acceptance ratios are set as $r_{s}=0.1$ and $r_{e}=0.01$. The number of iterations at each temperature is set to be equal to the number of total neighborhoods used (i.e $n r e p=5$ ). This implies that each neighborhood is sampled, on average, once at each temperature level if the neighborhoods are selected uniformly. We set the length of a single learning period $L P=5000$ and the minimum weight for each heuristic $w_{\min }=1 / n$. The total iteration count is set to different values based on the size of the problem instances. We set $K=100,000$ for the small instance Pn6, $K=500,000$ for the medium instance Pn29 (see section 6.2), and $K=2 \times 10^{6}$ for all the artificially generated instances (see section 6.4). 


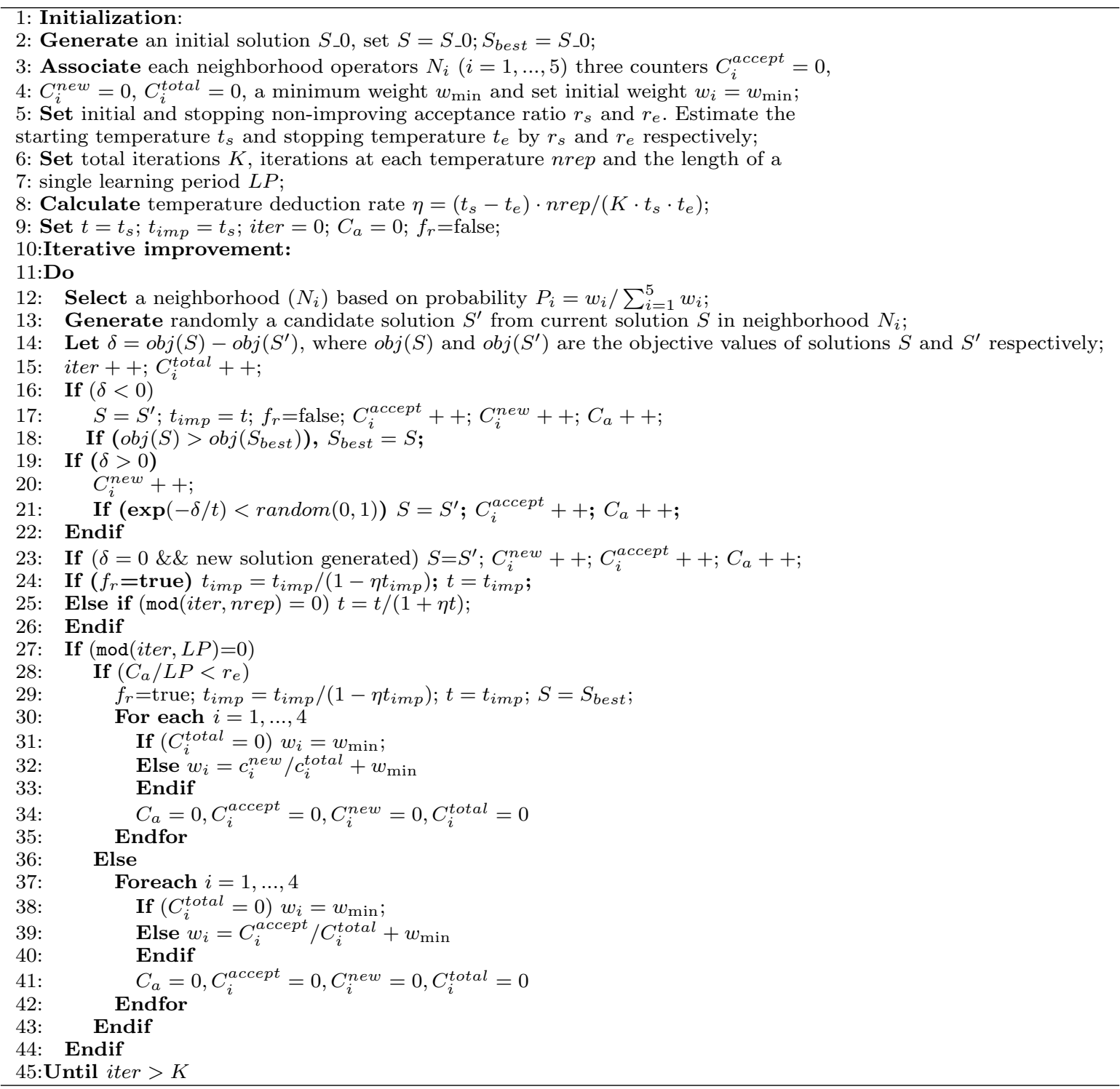

Fig. 2. Pseudo code of the proposed multiple neighborhood search algorithm (Bai et al., 2012).

\section{Extension: no clustering constraint}

As mentioned earlier, the inclusion of a cluster constraint (12) simplifies the problem in a sense that the search can concentrate on a smaller search space. However, the solution procedures proposed in this paper can be easily adapted to the problem without this cluster constraint. For the gradient approach, choosing both the best shelf and item will be based on function (13) only. For the multi-neighbourhood approach, two things need to change: a) feasibility check procedure will be relaxed accordingly. b) New neighourhood moves can be introduced which reallocate some of facings to another shelf. The overall framework and the other aspects of the algorithm will remain the same. This also highlights one of the advantages of the proposed 
multi-neighbourhood approach, which could adapt to the changes in a business environment. However, this may not be the case for some mathematical programming techniques.

\section{Numerical Examples}

In this section, we describe the empirical data available and then present some examples based on this data.

\subsection{Empirical input data}

We use empirical data on daily sales, product attributes and available shelf space obtained from a European supermarket chain. We focused on dry groceries which are delivered from the retailers' distribution center. Median sales per SKU are observed to be around 3.8 customer unit/week. The merchandising categories which were included in the datasets are similar to the categories reported in (Broekmeulen et al., 2007). The experiment contained SKUs (Stock-Keeping-Unit) from 44 stores. Usually, promotions are done in the stores using other shelf space allocations. Therefore, promotions are eliminated to obtain the true mean and the standard deviation of the weekly sales. The supermarket chain reported the shelf capacity for each store-SKU combination. Unfortunately, the chain did not have data on the exact position of the products on the shelves, nor did they report on the specific sales prices of the SKUs due to reasons of confidentiality.

Table 3. Problem instance with 6 items

\begin{tabular}{cccccccccccccc}
\hline & & & & & & \multicolumn{9}{c}{$\beta_{i k}$} \\
${ } } }$ & $h_{i}$ & $l_{i}$ & $p_{i}$ & $s_{i}^{\text {min }}$ & $s_{i}^{\max }$ & $\alpha_{i}$ & $\beta_{i}$ & 1 & 2 & 3 & 4 & 5 & 6 \\
\hline 1 & 143 & 47 & 6.62 & 3 & 10 & 45.80 & 0.88 & - & -0.001 & 0.008 & 0 & 0 & 0 \\
2 & 143 & 47 & 5.94 & 2 & 8 & 93.57 & 0.73 & 0.029 & - & 0.022 & 0 & 0 & 0 \\
3 & 150 & 50 & 4.59 & 3 & 8 & 42.59 & 0.88 & 0.016 & 0.026 & - & 0 & 0 & 0 \\
4 & 150 & 76 & 3.92 & 1 & 10 & 68.52 & 0.34 & 0 & 0 & 0 & - & 0 & 0 \\
5 & 143 & 47 & 7.93 & 1 & 6 & 22.74 & 0.80 & 0 & 0 & 0 & 0 & - & 0 \\
6 & 150 & 50 & 6.09 & 3 & 10 & 16.92 & 0.66 & 0 & 0 & 0 & 0 & 0 & - \\
\hline \multicolumn{3}{l}{ Shelf data: $m=3, L_{j}=500, H_{j}=\{150,300,150\}, \gamma_{j}=\{1.0,1.1,1.0\}$} \\
\hline
\end{tabular}




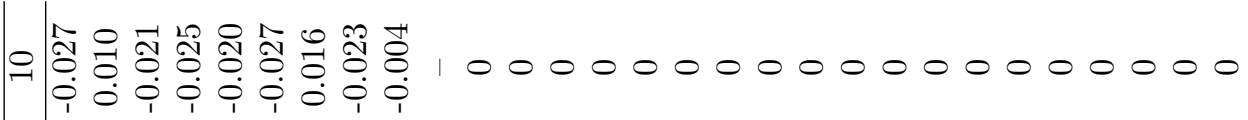

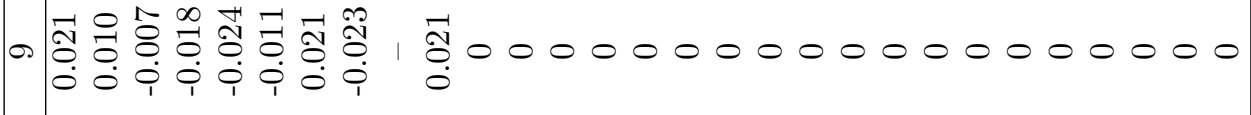

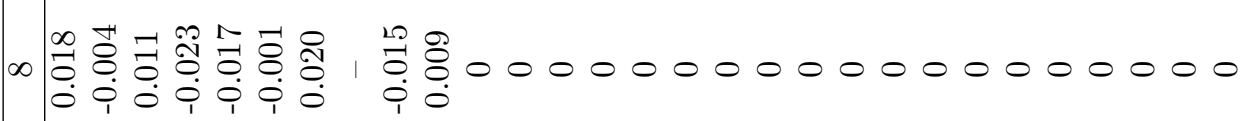

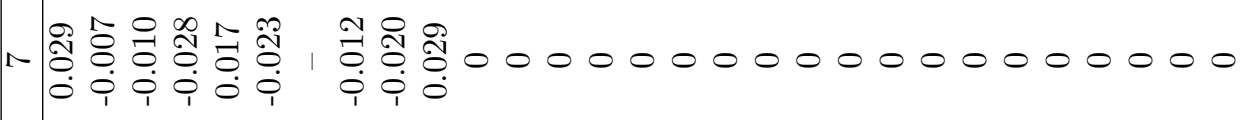
$\approx$

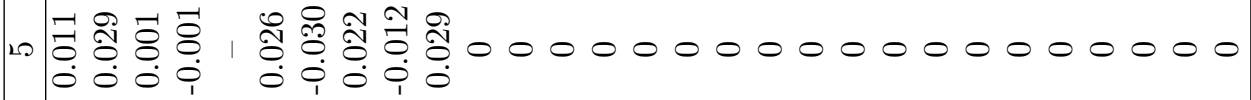

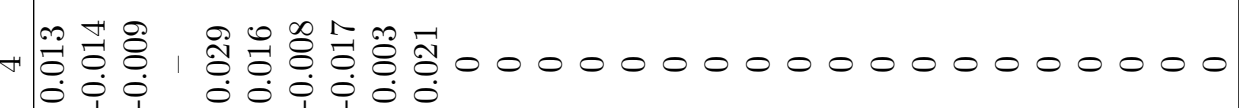
m:

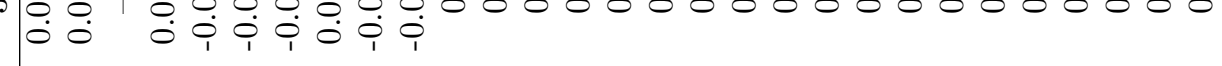
N N $\begin{array}{lll}0 \\ 0\end{array}$

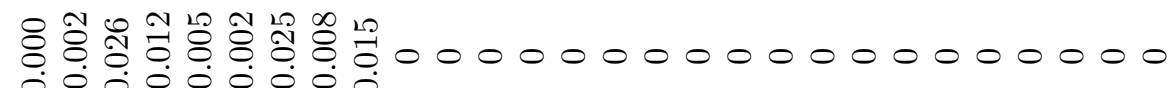
Oن

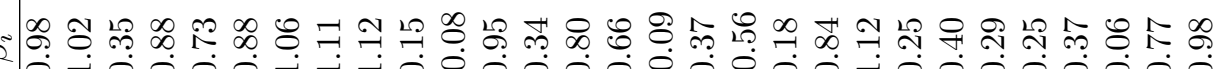

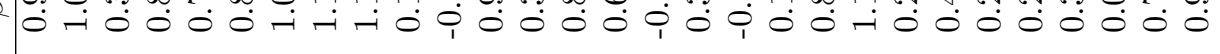

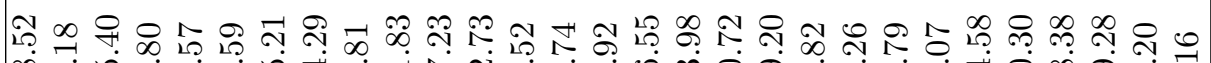

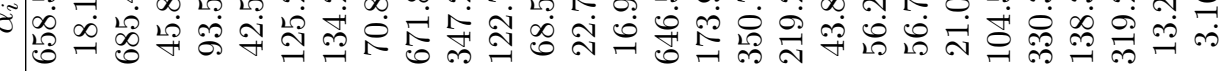

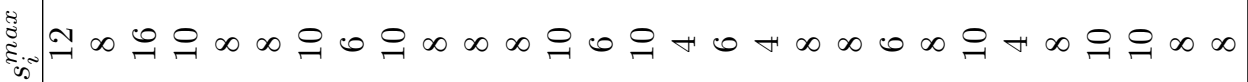

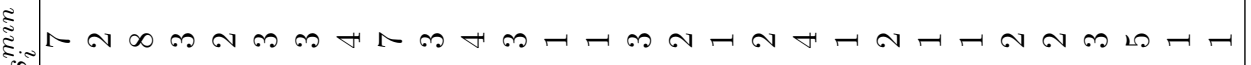

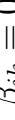

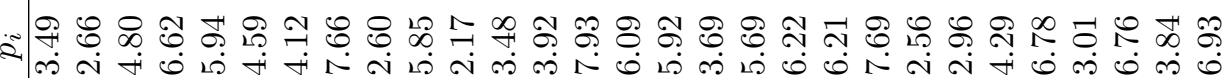

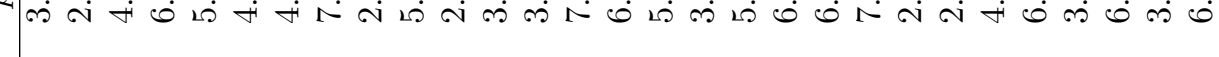

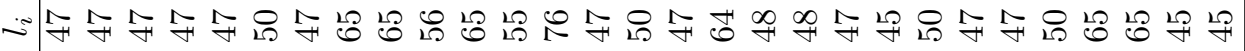

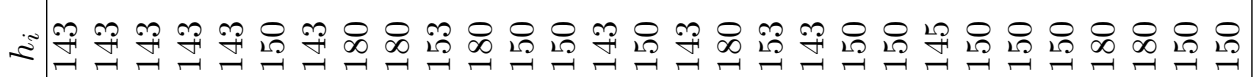
ค 


\subsection{Two case studies}

Due to data availability and commercial confidentiality limitations, only two instances, one small instance ( $m=3, n=6)$ and one medium-sized instance $(m=5, n=29)$, were extracted from the data. For simplicity, they are denoted as Pn6 and Pn29. Detailed information of these two instances is provided in the Tables 3 and 4 . The instances are based on a coffee category. Due to the limited data available, sales prices are drawn from the range $[2.0,8.0]$ with even probabilities and cross elasticities were uniformly sampled within $[-0.03,0.03]$ for the top 10 SKUs (in sales). The elasticities for the other SKUs are set to be zero. Direct space elasticities $\beta_{i}$ were estimated by simple linear regression based on the real-world data. As can be seen from Table 7 , most of direct space elasticities are in the range $[0,1]$. There are a few values outside this range though. A negative value means that a big displayed stock may leads to a decrease in demand (sometimes, a customer may associate poor quality or deficits with some large-stocked items). Some direct space elasticities are larger than 1 which means that allocating extra space to these items would lead to a larger increase in demand. Note that these estimated values are only valid within the corresponding facings ranges $\left[s_{i}^{\min }, s_{i}^{\max }\right]$. Once the allocated facings exceed this bound, the estimated direct elasticity values become invalid. All the other values were directly drawn from the real-world data.

Table 5. A comparison of solutions by different approaches for Pn6.

\begin{tabular}{|c|c|c|c|c|c|c|c|c|c|}
\hline \multirow[b]{2}{*}{ Item $_{i}$} & \multicolumn{3}{|c|}{ Gradient } & \multicolumn{3}{|c|}{ Multi-neighborhood } & \multicolumn{3}{|c|}{ Optimal Solution } \\
\hline & $x_{i}$ & $s_{i}$ & $z_{i}$ & $x_{i}$ & $s_{i}$ & $z_{i}$ & $x_{i}$ & $s_{i}$ & $z_{i}$ \\
\hline 1 & 7 & 7 & 0 & 5 & 10 & 1 & 5 & 10 & 1 \\
\hline 2 & 4 & 8 & 1 & 4 & 8 & 1 & 4 & 8 & 1 \\
\hline 3 & 8 & 8 & 2 & 8 & 8 & 0 & 8 & 8 & 0 \\
\hline 4 & 2 & 4 & 1 & 1 & 2 & 1 & 1 & 2 & 1 \\
\hline 5 & 3 & 6 & 1 & 6 & 6 & 2 & 6 & 6 & 2 \\
\hline 6 & 3 & 3 & 0 & 4 & 4 & 2 & 4 & 4 & 2 \\
\hline Objective (Best/Mean)* & \multicolumn{3}{|c|}{8188.97} & \multicolumn{3}{|c|}{$8975.50 / 8897.75$} & \multicolumn{3}{|c|}{8975.50} \\
\hline Deviation from Optimum & \multicolumn{3}{|c|}{$8.8 \%$} & \multicolumn{3}{|c|}{$0.9 \%$} & \multicolumn{3}{|c|}{-} \\
\hline Time (in sec.) & \multicolumn{3}{|c|}{$<0.1$} & \multicolumn{3}{|c|}{1.2} & \multicolumn{3}{|c|}{58.6} \\
\hline
\end{tabular}

*The best and mean objective by the multi-neighborhood algorithm were based on 10 independent runs. Both the gradient approach and the complete enumeration approach always obtained the same solution.

Tables 5 and 6 present a comparison of solutions obtained by the gradient approach, the multi-neighborhood approach and the complete enumeration approach. All the algorithms were run on a PC with a Pentium IV 1.8GHZ CPU and 2GB RAM. Due to its stochastic nature, the multi-neighborhood approach was run 20 times for each problem instance (with the best solution and average objective value over 20 runs being presented in the tables). From Table 5, it can be seen that the gradient approach is very fast. For the small 
Table 6. Solutions obtained by the gradient method and the multi-neighborhood approach for Pn29.

\begin{tabular}{|c|c|c|c|c|c|c|}
\hline \multirow[b]{2}{*}{ Item $_{i}$} & \multicolumn{3}{|c|}{ Gradient } & \multicolumn{3}{|c|}{ Multi-neighborhood (MN2) } \\
\hline & $x_{i}$ & $s_{i}$ & $z_{i}$ & $x_{i}$ & $s_{i}$ & $z_{i}$ \\
\hline 1 & 6 & 1 & 12 & 6 & 12 & 1 \\
\hline 2 & 8 & 3 & 8 & 2 & 2 & 3 \\
\hline 3 & 8 & 4 & 16 & 8 & 16 & 1 \\
\hline 4 & 10 & 2 & 10 & 10 & 10 & 2 \\
\hline 5 & 8 & 2 & 8 & 8 & 8 & 2 \\
\hline 6 & 3 & 3 & 3 & 3 & 3 & 3 \\
\hline 7 & 5 & 4 & 10 & 10 & 10 & 2 \\
\hline 8 & 6 & 0 & 6 & 6 & 6 & 1 \\
\hline 9 & 10 & 1 & 10 & 10 & 10 & 4 \\
\hline 10 & 4 & 1 & 8 & 3 & 3 & 0 \\
\hline 11 & 4 & 0 & 4 & 4 & 4 & 4 \\
\hline 12 & 8 & 3 & 8 & 8 & 8 & 3 \\
\hline 13 & 3 & 2 & 3 & 3 & 3 & 0 \\
\hline 14 & 6 & 2 & 6 & 6 & 6 & 2 \\
\hline 15 & 7 & 3 & 7 & 5 & 10 & 4 \\
\hline 16 & 2 & 2 & 2 & 1 & 2 & 1 \\
\hline 17 & 6 & 0 & 6 & 6 & 6 & 0 \\
\hline 18 & 2 & 4 & 2 & 1 & 2 & 1 \\
\hline 19 & 4 & 4 & 8 & 8 & 8 & 3 \\
\hline 20 & 4 & 1 & 8 & 8 & 8 & 0 \\
\hline 21 & 6 & 3 & 6 & 3 & 6 & 1 \\
\hline 22 & 1 & 2 & 1 & 3 & 3 & 3 \\
\hline 23 & 1 & 2 & 1 & 1 & 1 & 0 \\
\hline 24 & 4 & 0 & 4 & 2 & 4 & 1 \\
\hline 25 & 4 & 4 & 8 & 4 & 8 & 1 \\
\hline 26 & 5 & 0 & 5 & 4 & 4 & 4 \\
\hline 27 & 5 & 4 & 5 & 6 & 6 & 0 \\
\hline 28 & 1 & 2 & 1 & 8 & 8 & 3 \\
\hline 29 & 3 & 4 & 6 & 4 & 8 & 4 \\
\hline Objectve (Best/mean)* & \multicolumn{3}{|c|}{97134.70} & \multicolumn{3}{|c|}{$110640.14 / 110007.113$} \\
\hline Time (in sec.) & \multicolumn{3}{|c|}{$<0.5$} & \multicolumn{3}{|c|}{43.4} \\
\hline
\end{tabular}

*The best and mean objective by the multi-neighborhood algorithm were based on 20 independent runs. The gradient approach always returned the same solution.

instance Pn6, it finds a solution in less than 0.1 seconds with an objective value that is $8.8 \%$ away from the optimum. In contrast, the complete enumeration is quite slow, with a computational time of almost a minute. For Pn29, the complete enumeration approach (embedded with some simple bounding heuristics) failed even to find a feasible solution after 12 hours of computational time. The multi-neighborhood approach seems to provide an appropriate compromise between the solution quality and the computational time. On average, among 20 runs, it obtains a solution that is only $0.8 \%$ away from the optimum with much less computational time. For the small instance Pn6, the multi-neighborhood approach is able to find the opti- 
mal solution among 20 runs. For the instance Pn29, the multi- neighborhood approach could obtain $12.3 \%$ $\left(=\frac{110640.14-97134.70}{97134.70}\right)$ improvement over the gradient approach in terms of the objective function. Nevertheless, the multi-neighborhood approach consumes much more (but still reasonable) CPU time. A graphical representation of the best solution by the multi-neighborhood approach is given in the Fig. 3 .
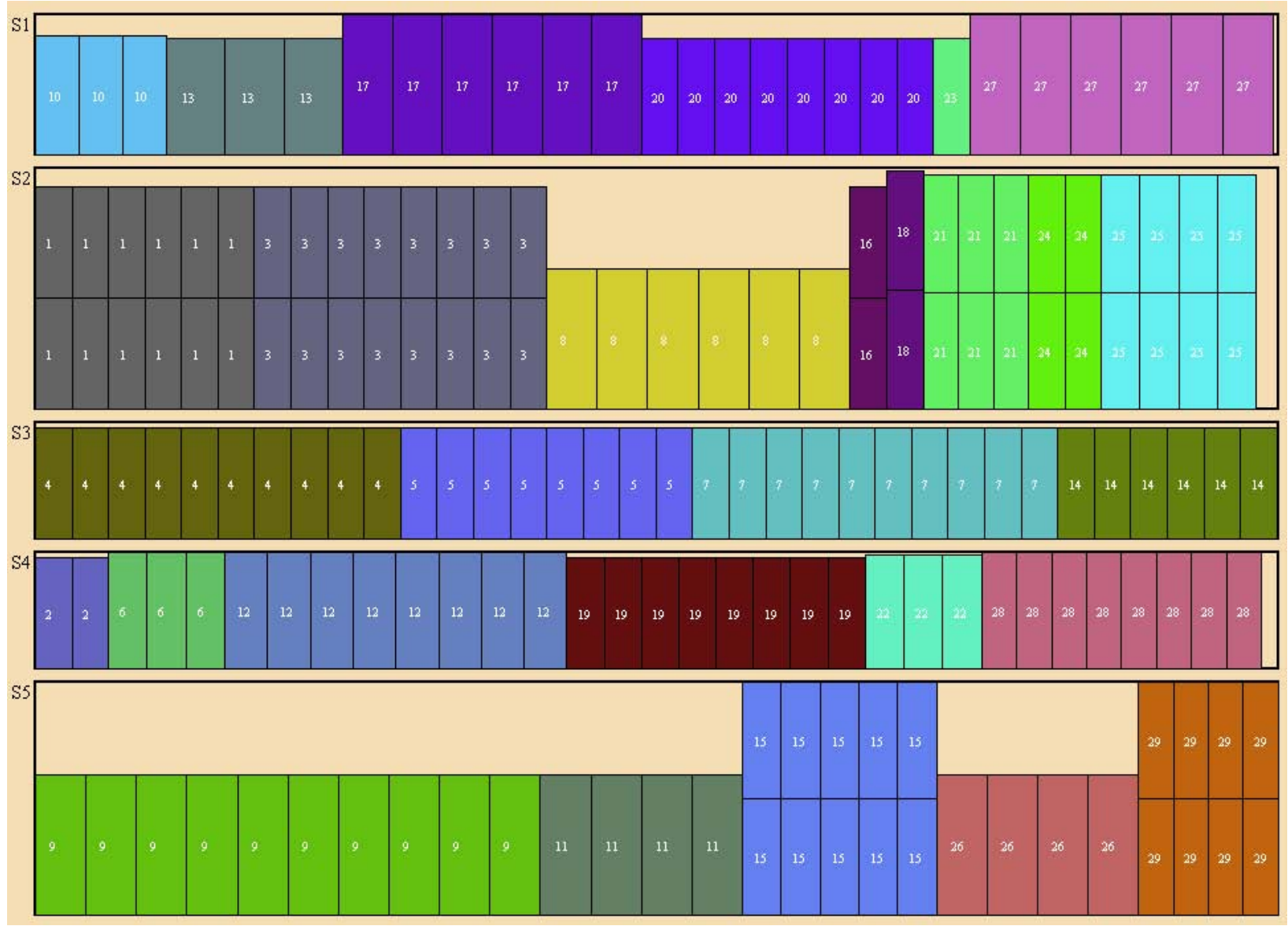

Fig. 3. The best solution obtained by the multi-neighborhood approach out of 20 runs

\subsection{Sensitivity Analysis}

Shelf space Although shelf space is an expensive resource, in some cases, retailers may be able to invest more in space in order to increase sales. The question that retailers are interested in is how much extra sales can be realized by investing in more shelf space. The small instance is considered here since its optimal solution can be found reasonably quickly by the complete enumeration method. Fig. 4 plots the relationship between the percentage of increased sales and the increase in shelf length (the shelves' height dimensions are the same). 
It can be seen that increasing shelf length has a positive effect on the sales. However, this relationship is extremely nonlinear. For example, when the shelf length is increased from 300 to 340, the sales increased by almost 15\%. However, expanding the shelf length from 780 to 1000 does not increase sales at all. In general, when the shelf space is very limited, an increase in the shelf space has a larger impact on the sales than when the shelf space is already large. When the shelf length reaches over 1400, it no longer has an impact on sales. This is partially due to the diminishing return demand function we used. In addition, the number of facings allocated to the items may reach the maximum allowed upper bounds, which define the valid ranges of the demand function.

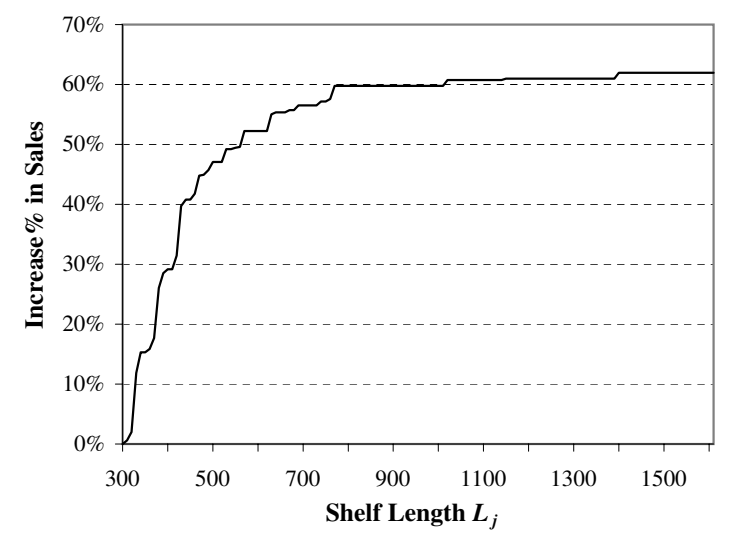

Fig. 4. The impact of shelf space over sales.

Sensitivity of the parameter estimation error A number of parameters were estimated in the problem model. For example, the scale coefficient $\left(\alpha_{i}\right)$, the space elasticity $\left(\beta_{i}\right)$, and the cross elasticities $\left(\beta_{i k}\right)$. This could introduce some estimation errors into the model. In this section, we analyze the effect of these estimation errors over the objective function. The small instance was used again due to its known optimal objective. The sensitivity analysis method is similar to the approach used in (Borin and Farris, 1995). For each estimated parameter set $\left(\alpha_{i}, \beta_{i}\right.$, or $\left.\beta_{i k}\right)$, a random error is added to each element of the considered parameter set. The errors were sampled from a normal distribution with mean values set to 0 . The errors have mean 0 , while the resulting parameters have means to be the true values and increasingly larger standard deviations. Let $X \%$ be the mean absolute percent error from the true values. The enumeration search was again carried out to obtain the global optimal objective values for both the original instance (without error) and the instance with parameter errors. Let $M_{\text {orig }}$ and $M_{\text {error }}$ be the corresponding optimal objective values. A relative objective 
error, $M_{e} \%$, was calculated by $\left|M_{\text {orig }}-M_{\text {error }}\right| / M_{\text {orig }}$. For each parameter set, this process was repeated 50 times. The average relative objective errors are presented in Table 7.

Table 7. Average impact of errors in parameter estimation.

\begin{tabular}{|c|c|c|c|c|c|}
\hline \multicolumn{2}{|c|}{$\alpha_{i}$} & \multicolumn{2}{|c|}{$\overline{\beta_{i}}$} & \multicolumn{2}{|c|}{$\beta_{i k}$} \\
\hline$X \%$ & $M_{e} \%$ & $X \%$ & $M_{e} \%$ & $X \%$ & $M_{e} \%$ \\
\hline $2.7 \%$ & $1.28 \%$ & $2.5 \%$ & $2.31 \%$ & $3.2 \%$ & $1.52 \%$ \\
\hline $5.4 \%$ & $2.57 \%$ & $5.6 \%$ & $4.61 \%$ & $6.4 \%$ & $1.49 \%$ \\
\hline $7.6 \%$ & $3.80 \%$ & $7.4 \%$ & $7.59 \%$ & $9.6 \%$ & $1.53 \%$ \\
\hline $10.6 \%$ & $5.48 \%$ & $10.4 \%$ & $7.03 \%$ & $12.9 \%$ & $1.63 \%$ \\
\hline $14.2 \%$ & $6.61 \%$ & $12.3 \%$ & $9.37 \%$ & $16.3 \%$ & $1.54 \%$ \\
\hline $16.8 \%$ & $7.14 \%$ & $15.2 \%$ & $11.67 \%$ & $18.9 \%$ & $1.82 \%$ \\
\hline $17.7 \%$ & $13.76 \%$ & $16.0 \%$ & $16.94 \%$ & $23.0 \%$ & $1.82 \%$ \\
\hline $21.2 \%$ & $10.64 \%$ & $17.4 \%$ & $12.69 \%$ & $26.0 \%$ & $1.67 \%$ \\
\hline $22.4 \%$ & $12.13 \%$ & $20.5 \%$ & $12.19 \%$ & $27.0 \%$ & $1.83 \%$ \\
\hline
\end{tabular}

$X \%$ : Mean absolute percent error in parameter estimation $M_{e} \%$ : Mean percent deviation from optima

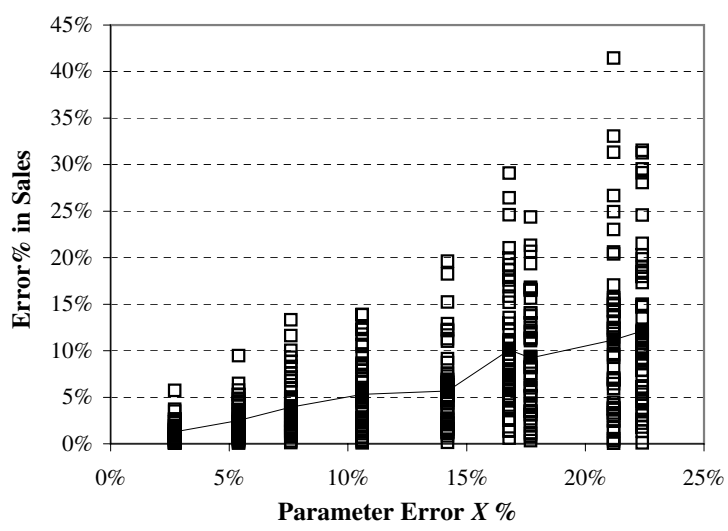

Fig. 5. Distribution of errors in sales with respect to errors in $\alpha_{i}$

Figs. 5, 6, 7 plot the distributions of the resulted errors in sales with respect to the errors in three parameter sets: $\alpha_{i}, \beta_{i}$, and $\beta_{i k}$. Each square box represents a given percentage error in sales. Hence, areas crowded with square boxes mean more values appearing in that range. The solid line plots the trend of the average relative error in sales across different parameter errors. The evidence suggests that the model is reasonably robust. In general, average errors in sales range from $1.28 \%$ to less than $17 \%$. Larger errors in parameter estimation generally result in larger errors in sales prediction by the model, except for the cross elasticity. Within three parameter sets, the errors in $\alpha_{i}$ and $\beta_{i}$ lead to a larger errors in sales than the $\beta_{i k}$. 


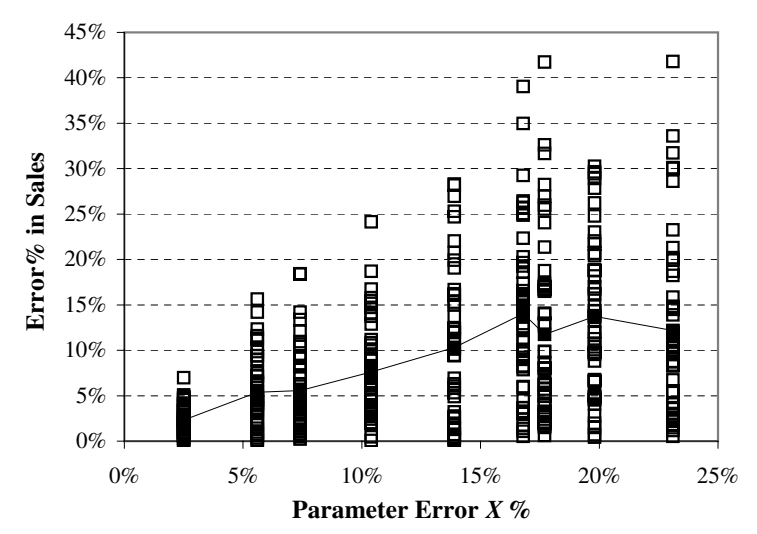

Fig. 6. Distribution of errors in sales with respect to the errors in $\beta_{i}$

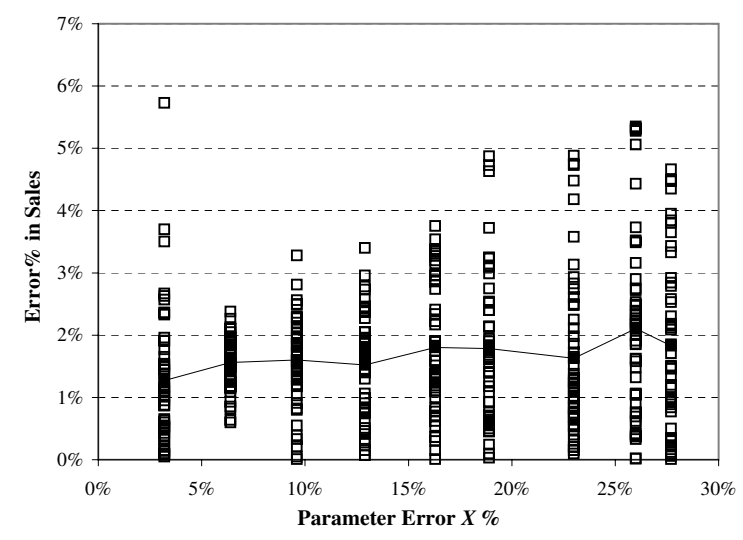

Fig. 7. Distribution of errors in sales with respect to the errors in $\beta_{i k}$

Cross elasticities have least impact on the sales. This is probably due to the fact that their values are much smaller compared with $\alpha_{i}$ and $\beta_{i}$.

\subsection{Larger instances}

In order to fully test the model and the solution methods, we generated two artificial data sets (Set1 and Set2), each of which is comprised by 20 larger instances. The parameters that are used to create these instances are the same to those used by Hwang et al. (2009). Each instance in Set1 contains 50 items and 5 shelves (denoted as n50m5_01-n50m5_10). Instances in Set2 have 100 items and 10 shelves (denoted as n1000m10_01n100m10_10). These instances are publicly available for download from http://www.cs.nott.ac.uk/ rzb/files/2dssa-instances.zip. 
Two versions of the simulated annealing hyper-heuristics were implemented and tested. In the first version (denoted as MN1), the neighborhoods are uniformly selected and hence does not have any adaptation. The second version is the one that we described in Fig. 2 where the probabilities of neighborhood calls are adaptively tuned during the search. All the other parameters for MN1 and MN2 are the same. In addition, we also implemented a first-decent variable neighbourhood search (VNS), consisting of two repetitive phases, local search phase and perturbation phase. The neighborhoods N1, N2, N3, N4 described in section 4.2.1 were used as the first-descent local search neighborhoods and N5 was used as a perturbation method. The VNS algorithm successively searches through each of the local search neighborhoods (i.e. N1-N4) until it gets stuck at a local optimum. A perturbation is then applied to the current solution using neighborhood function N5 before the next iteration of the local search phase.

The following parameter settings were used: for both MN1 and MN2, we set $K=2 \times 10^{6}, L P=5000$, which corresponds to around 280 seconds computational time for Set1 instances and 600 seconds for Set2 instances on the same machine that we used in the previous experiments. For VNS, computational time limit was set to 280 seconds for Set1 instances and 600 seconds for Set2 instances. Therefore, all the algorithms used approximately similar amount of computational time. Each of the 20 instances was solved by each of these algorithm (MN1, MN2, VNS) 20 times from different random seeds. 


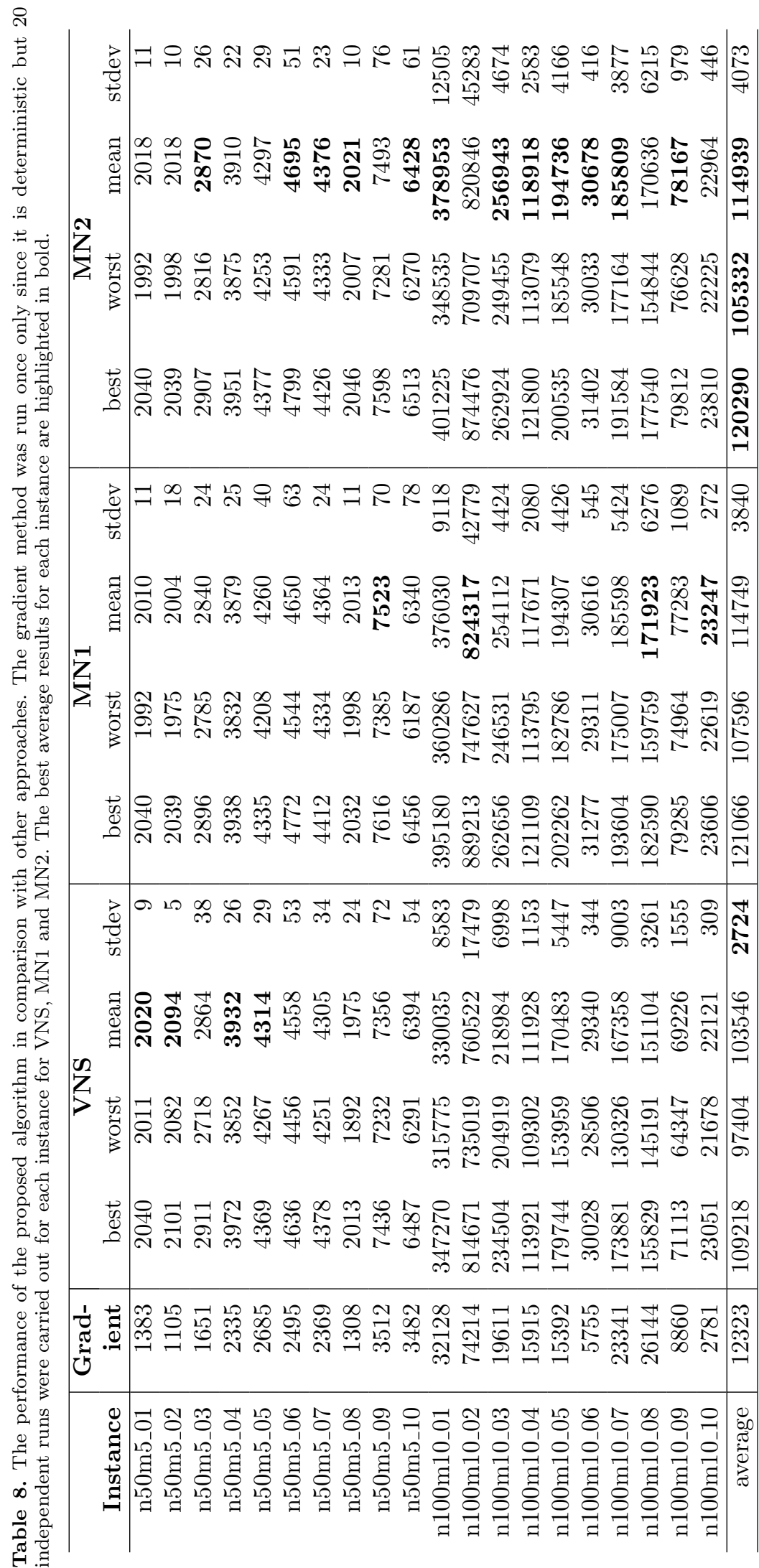


Table 8 gives the computational results for these 20 instances by the gradient method, the variable neighborhood search (VNS), the multi-neighborhood approach with random neighborhood selection (MN1), multi-neighborhood approach with adaptive neighborhood selection (MN2). A few observations can be made from the results; 1) The gradient method performed poorly, particularly for instances with 100 items and 10 shelves. 2) The variable neighbourhood search (VNS) does well for Set1 instances but are significantly inferior to MN1 and MN2 for instances in Set2. 3) In terms of average objective values, the hyper-heuristic approach with adaptive neighbourhood selection (MN2) performed generally better than the random neighbourhood selection (MN1). Nevertheless, the mean results by MN1 were better for 4 instances (e.g. n50_08, n100_02, n100_08, n100_10).

The two-tailed student's t-tests were also carried out to find out whether the proposed hyper-heuristic method (MN2) is statistically different from VNS or MN1. Table 9 presents the probabilities of the test results. We can see that, at 5\% confidence level, MN2 is significantly different from VNS for every instance. From both Table 8 and Table 9, we can conclude that MN2 is significantly better than VNS except for 3 out 20 instances (n50m5_07, n50m5_08, and n50m5_10), for which VNS performed better. The differences in performance between MN1 and MN2 are not as obvious but overall MN2 is slightly better. For 8 out of 20 instances, there is no significant difference between them. For the remaining 12 instances, MN2 is significantly better than MN1 for 11 instances and MN1 outperformed MN2 statistically for 1 instance, n100m10_10.

Table 9. The probability results of the student's t-tests between MN2, VNS and MN1.

\begin{tabular}{l|c|c|l|c|c}
\hline Set1 & MN2 vs VNS & MN2 vs MN1 & Set2 & MN2 vs VNS & MN2 vs MN1 \\
\hline n50m5_01 & $2.4 \%$ & $4.2 \%$ & n100m10_01 & $0.0 \%$ & $40.7 \%$ \\
n50m5_02 & $0.0 \%$ & $0.8 \%$ & n100m10_02 & $0.0 \%$ & $80.7 \%$ \\
n50m5_03 & $4.5 \%$ & $0.2 \%$ & n100m10_03 & $0.0 \%$ & $3.6 \%$ \\
n50m5_04 & $0.0 \%$ & $0.0 \%$ & n100m10_04 & $0.0 \%$ & $2.9 \%$ \\
n50m5_05 & $0.0 \%$ & $0.2 \%$ & n100m10_05 & $0.0 \%$ & $78.4 \%$ \\
n50m5_06 & $0.0 \%$ & $1.1 \%$ & n100m10_06 & $0.0 \%$ & $62.5 \%$ \\
n50m5_07 & $0.0 \%$ & $10.2 \%$ & n100m10_07 & $0.0 \%$ & $88.4 \%$ \\
n50m5_08 & $0.0 \%$ & $3.2 \%$ & n100m10_08 & $0.0 \%$ & $52.3 \%$ \\
n50m5_09 & $0.0 \%$ & $18.8 \%$ & n100m10_09 & $0.0 \%$ & $1.4 \%$ \\
n50m5_10 & $1.7 \%$ & $0.0 \%$ & n100m10_10 & $0.0 \%$ & $4.8 \%$ \\
\hline
\end{tabular}

\subsection{Adaptation of neighborhood selection}

In our proposed algorithm, the selection of neighborhoods are based on an online learning mechanism. As we discussed in the previous section, this online selection mechanism provides a slightly better overall 


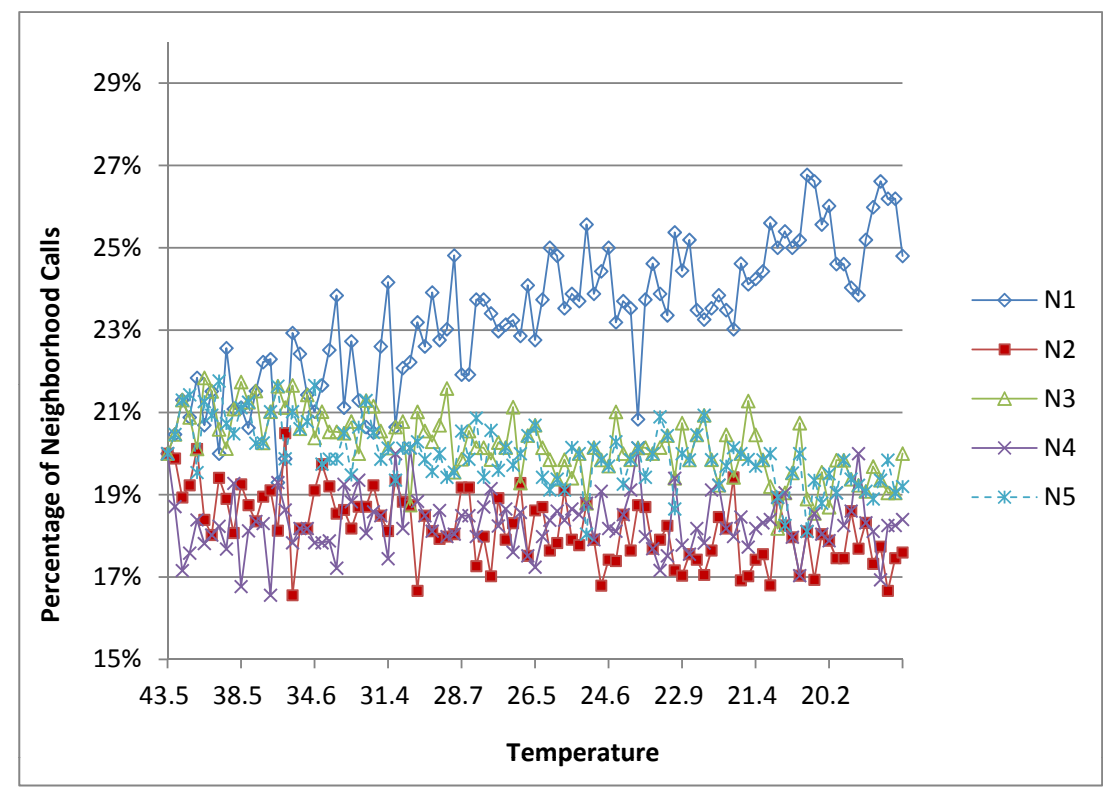

Fig. 8. Adaptation of neighbourhood calls at different annealing temperatures (For instance n50m5_01. Distributions for other instances are similar.)

performance than the random uniform neighborhood selection for most instances. Fig. 8 shows a typical dynamic adaptation process of different neighbourhood calls over different annealing temperatures. It can be observed that, although the probabilities with which neighborhoods were chosen are the same at the beginning of the search, they are changed for different temperatures during the search. Overall, the first neighbourhood, N1, was selected more than the other neighborhoods. This trend intensifies when the annealing temperature decreases.

\section{Conclusions}

In this paper, we have presented a two-dimensional shelf space allocation model. The contributions of the paper are two folds: 1) rather than focusing on a single dimension, shelf length, the proposed shelf space allocation model adds the height dimension to the shelf space allocation decisions. As such, the new model is much more realistic than one-dimensional models. 2) An efficient simulated annealing hyper-heuristic approach is proposed for this $2 \mathrm{D}$ shelf space allocation problem. This approach can flexibly be adapted to other types of shelf space allocation problems (e.g. three-dimensional versions) thanks to the increased generality of hyper-heuristics compared to other heuristic methods. The performance of the algorithm were tested based on 2 real-world instances and 20 artificial instances. 
We have showed via a small example used in a previous study, that explicitly taking into account the height dimension will improve shelf space utilization and the resulting sales. Sensitivity analysis has shown the benefits of the proposed algorithm: if one has allocated limited shelf space to an SKU, a shelf space increase will have a relatively large impact on sales than if one already has abundant shelf space allocated. In addition, the shelf space allocation model is fairly robust against input parameter errors.

\section{References}

R. Bai and G. Kendall. An investigation of automated planograms using a simulated annealing based hyper-heuristics. In

T. Ibaraki, K. Nonobe, and M. Yagiura, editors, Metaheuristics: Progress as Real Problem Solvers, volume 32 of Operations Research/Computer Science Interfaces, pages 87-108. Springer, 2005.

R. Bai and G. Kendall. A model for fresh produce shelf space allocation and inventory management with freshness condition dependent demand. INFORMS Journal on Computing, 20(1):78-85, 2008.

R. Bai, E. K. Burke, and G. Kendall. Heuristic, meta-heuristic and hyper-heuristic approaches for fresh produce inventory control and shelf space allocation. Journal of the Operational Research Society, 59:1387-1397, 2008.

R. Bai, J. Blazewicz, E. K. Burke, G. Kendall, and B. McCollum. A simulated annealing hyper-heuristic methodology for flexible decision support. 4OR - A Quarterly Journal of Operations Research, 10:43-66, 2012.

N. Borin and P. Farris. A sensitivity analysis of retailer shelf management models. Journal of Retailing, 71(2):153-171, 1995.

N. Borin, P. W. Farris, and J. R. Freeland. A model for determining retail product category assortment and shelf space allocation. Decision Sciences, 25(3):359-384, 1994

R. A. C. M. Broekmeulen, J. C. Fransoo, and K. H. van Donselaar T. van Woensel. Shelf space excesses and shortages in grocery retail stores. Technical report, Eindhoven University of Technology, The Netherlands, 2007.

E. K. Burke, E. Hart, G. Kendall, J. Newall, P. Ross, and S. Schulenburg. Hyper-heuristics: An emerging direction in modern search technology. In F. Glover and G. Kochenberger, editors, Handbook of Metaheuristics, pages 457-474. Kluwer, 2003 a.

E. K. Burke, G. Kendall, and E. Soubeiga. A tabu-search hyperheuristic for timetabling and rostering. Journal of Heuristics, 9(6):451-470, 2003b.

E. K. Burke, S. Petrovic, and R. Qu. Case based heuristic selection for timetabling problems. Journal of Scheduling, 9(2): $115-132,2006$.

E. K. Burke, B. McCollum, A. Meisels, S. Petrovic, and R. Qu. A graph-based hyper heuristic for educational timetabling problems. European Journal of Operational Research, 176(1):177-192, 2007.

M. Corstjens and P. Doyle. A model for optimising retail space allocations. Management Science, 1981.

K. Cox. The effect of shelf space upon sales of branded products. Journal of Marketing Research, 7:55-58, 1970.

R. Curhan. The relationship between space and unit sales in supermarkets. Journal of Marketing Research, 9:406-412, 1972.

R. C. Curhan. Shelf space allocation and profit maximization in mass retailing. Journal of Marketing, 37:54-60, 1973. 
K. A. Dowsland, E. Soubeiga, and E. K. Burke. A simulated annealing based hyperheuristic for determining shipper sizes for storage and transportation. European Journal of Operational Research, 179(3):759-774, 2007.

X. Dreze, S. J. Hoch, and M. E. Purk. Shelf management and space elasticity. Journal of Retailing, 70(4):301-326, 1994.

F. Glover and G. A. Kochenberger, editors. Handbook of Metaheuristics. Springer, 2003.

David Goldberg. Genetic Algorithms in Search, Optimization and Machine Learning. Addison Wesley, 1989.

E. Hart, P. Ross, and J. A. Nelson. Solving a real-world problem using an evolving heuristically driven schedule builder. Evolutionary Computing, 6(1):61-80, 1998.

H. Hwang, B. Choi, and M.-J. Lee. A model for shelf space allocation and inventory control considering location and inventory level effects on demand. International Journal of Production Economics, 97(2):185-195, 2005.

Hark Hwang, Bum Choi, and Grimi Lee. A genetic algorithm approach to an integrated problem of shelf space design and item allocation. Computers \& Industrial Engineering, 56:809-820, 2009.

J. Kotzan and R. Evanson. Responsiveness of drug store sales to shelf space allocations. Journal of Marketing Research, 6: 465-469, 1969.

A. Lim, B. Rodrigues, and X. Zhang. Metaheuristics with local search techniques for retail shelf-space optimization. Management Science, 50(1):117-131, 2004.

Chase C. Murray, Debabrata Talukdar, and Abhijit Gosavi. Joint optimization of product price, display orientation and shelf-space allocation in retail category management. Journal of Retailing, 86:125C136, 2010.

P. Ross. Hyper-heuristics. In E. K. Burke and G. Kendall, editors, Search Methodologies: Introductory Tutorials in Optimization and Decision Support Techniques, chapter 17, pages 529-556. Springer, 2005.

K. Sastry, D. Goldberg, and G. Kendall. Genetic algorithms. In E.K. Burke and G. Kendall, editors, Search Methodologies: Introductory Tutorials in Optimization and Decision Support Techniques, pages 97-125. Springer, 2005.

T. Urban. An inventory-theoretic approach to product assortment and shelf-space allocation. Journal of Retailing, 74(1):15-35, 1998.

T. Van Woensel, R.A.C.M. Broekmeulen, K.H. van Donselaar, and J.C. Fransoo. Planogram integrity: a serious issue. ECR Journal, 6:4-5, 2006.

M.-H. Yang. An efficient algorithm to allocate shelf space. European Journal of Operational Research, 131:107-118, 2001.

F. Zufryden. A dynamic programming approach for product selection and supermarket shelf-space allocation. Journal of Operations Research Society, 37(4):413-422, 1986. 